\title{
RELAÇÃO ENTRE A MOBILIDADE DO TORNOZELO E PÉ E A MAGNITUDE DA FORÇA VERTICAL DE REAÇÃO DO SOLO
}

\author{
VIANNA DL ${ }^{1}$ E GREVE JMD ${ }^{2}$ \\ ${ }^{1}$ Universidade Paulista; Faculdades Metropolitanas Unidas, São Paulo, SP - Brasil \\ ${ }^{2}$ Laboratório de estudo do movimento, Instituto de Ortopedia e Tramatologia, Faculdade de Medicina, Universidade de \\ São Paulo, SP - Brasil \\ Correspondência para: Denise Loureiro Vianna, Rua Carlos Vicari, 124, Bairro: Pompéia, CEP : 05033-070, São Paulo, SP - \\ Brasil, e-mail: dlvianna@globo.com
}

Recebido: 09/11/2005 - Aceito: 07/06/2005

\begin{abstract}
RESUMO
Objetivo: Verificar a relação entre a mobilidade do tornozelo e do pé, e o pico da força vertical de reação do solo, considerada como porcentagem do peso corporal, gerada durante a fase de apoio da marcha. Métodos: foram estudados pés normais do

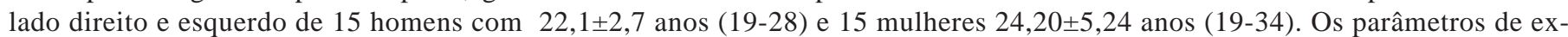
clusão foram: deformidades nos pés, doenças ou traumas, que pudessem acometer o sistema musculoesquelético e a marcha. A mobilidade do tornozelo e dos pés foi obtida através da goniometria da flexão plantar, dorsiflexão, extensão do hálux e extensão dos dedos, o pico da força vertical de reação do solo FRS, foi obtido pela baropodometria computadorizada do sistema FSCAN . A correlação entre ambas foi feita pelo teste estatístico de Spearman. Resultados: os indivíduos do grupo masculino apresentaram menores valores de mobilidade, e maiores valores do pico da força vertical de reação do solo, quando comparados com o grupo feminino. Não houve diferença entre os pés direito e esquerdo. No sexo feminino foi encontrada correlação negativa estatisticamente significante entre os valores da flexão plantar e a força vertical, e entre os valores da extensão dos dedos e a foça vertical. No sexo masculino, houve correlação negativa estatisticamente significante entre os valores da dorsiflexão e a força vertical. Entre os demais valores não foi encontrada correlação significante. Conclusão: Há relação entre a mobilidade e a força vertical gerada durante a marcha.
\end{abstract}

Palavras-chave: pé, mobilidade, força de reação do solo.

\section{ABSTRACT \\ Relationship Between Ankle and Foot Mobility and the Magnitude of the Vertical Ground Reaction Force}

Objective: To investigate the relationship between ankle and foot mobility and the peak of the vertical ground reaction force, as a percentage of body weight, generated during the gait stance phase. Method: Fifteen men with mean age of $22.1 \pm 2.7$ years (range: 19-28) and fifteen women with mean age of $24.20 \pm 5.24$ years (range: 19-34) with normal feet were studied. The exclusion criteria were foot deformities or a history of trauma or diseases that might have harmed both the musculoskeletal system and gait pattern. The ankle and foot mobility was obtained by means of goniometry on the plantar flexion, dorsiflexion, hallux extension and toe extension. The peak of the vertical ground reaction force was obtained by baropodometry using the FSCAN ${ }^{\mathrm{TM}}$ system. The Spearman statistical test was used to identify correlations. Results: Males presented lower mobility values and higher peak values for the vertical ground reaction force, in comparison with females. There was no difference between the right and left foot. For females, there were statistically significant negative correlations between the values for plantar flexion and vertical force, and between the values for toe extension and vertical force. For males, there were statistically significant negative correlations between the values for dorsiflexion and vertical force. Among the remaining values, no significant correlation was found. Conclusion: There was a relationship between mobility and the vertical force generated during gait.

Key words: foot, mobility, ground reaction force. 


\section{INTRODUÇÃO}

O pé humano constitui a base de apoio e propulsão para a marcha, sendo considerado um amortecedor dinâmico capaz de suportar, sem lesões, as cargas fisiológicas nele impostas $^{1,2}$. Esta capacidade se deve ao arranjo anatômico dos ossos, ligamentos e músculos, e dinamicamente, a adequada cinemática das diferentes articulações. Os movimentos dos pés são responsáveis pela absorção dos impactos, manutenção do equilíbrio e distribuição das forças ${ }^{3}$. Na literatura é possível encontrar estudos abordando a relação entre as alterações da cinemática dos pés e as forças plantares, em diferentes aspectos. Foi observado que o movimento de extensão dos dedos que ocorre na fase final do apoio, durante a marcha, é um importante evento para tornar o pé uma alavanca rígida, uma vez que ao tracionar a aponeurose plantar, esta se torna um reforço do arco plantar ${ }^{3,4,5}$. A presença da extensão dos dedos, durante a marcha também contribui para a redução da pressão plantar da região do antepé, uma vez que permite que os dedos mantenham o contato com o solo, aumentando a superfície de contato ${ }^{6}$. Pacientes com limitação da amplitude articular de extensão do hálux, tendem a apresentar aumento da pressão plantar no antepé, principalmente sob a região do primeiro metatarso ${ }^{7}$. Foi observado ainda, que a redução da mobilidade articular do tornozelo de indivíduos com artrodese nesta articulação, promove alterações na magnitude das cargas plantares, e na dinâmica da progressão da força vertical nas diferentes regiões dos pés ${ }^{8}$.

As alterações da cinemática normal dos pés também podem estar relacionadas com o surgimento de complicações em certas doenças. Estudos apontam que a limitação da mobilidade da articulação subtalar, têm relação com o aparecimento das úlceras plantares ${ }^{5,9}$, sendo esta questão bastante investigada nas áreas ligadas ao atendimento dos pacientes em situações de risco, como nos pés neuropáticos ${ }^{10,11,12,13}$. A redução da mobilidade articular também foi apontada como possível causa de dor nos atletas com pés cavos, quando comparados àqueles com os pés planos, fato que possivelmente se deve a maior rigidez apresentada pelos pés cavos, levando a menor deformação e absorção dos choques ${ }^{14}$. Observou-se ainda que na presença de limitações adquiridas da mobilidade, como nas cirurgias de artrodese, impõe uma dinâmica anormal nos pé com sobrecarga ${ }^{8}$ ou mesmo compensação das demais articulações ${ }^{15}$.

Atualmente observa-se uma crescente redução da mobilidade fisiológica dos pés, causada possivelmente pelo uso de calçados e pelo sedentarismo. Entre os jovens é possível observar aqueles que já apresentam considerável redução da amplitude de movimento dos dedos dos pés, e até mesmo deformidades, que outrora ocorriam, em sua maioria, em idades mais avançadas. Estas alterações certamente vão acarretar alguma alteração na função dos pés. Ao observar a cinética da marcha, o pé é a primeira estrutura do corpo a entrar em contato com o solo, e, portanto o primeiro a receber a força vertical gerada no contato, e assim transmitila para os demais segmentos. A interação da mobilidade dos pés e as forças envolvidas é um tema de grande abrangência não somente na presença da doença, mas na sua prevenção, onde o fisioterapeuta poderá atuar muitas vezes com a sua melhor ferramenta de trabalho, a cinesioterapia.

O objetivo deste trabalho foi verificar se existe correlação entre a mobilidade do tornozelo e pé, de homens e mulheres, e o pico da força vertical de reação do solo FRS, na fase de apoio da marcha utilizando os recursos da goniometria e do sistema FSCAN ${ }^{\mathrm{R}}$.

\section{MATERIAL E MÉTODOS}

Foram estudados os pés direito e esquerdo de 15 homens com idade média 22,1( $\pm 2,7)$ anos, mínimo 19 e máximo 28 anos e 15 mulheres com idade média de 24,20( $\pm 5,24)$ anos, mínima 19 e máxima 34 anos. Os critérios de inclusão foram: adulto jovem (18-40 anos), sem deformidades nos pés, ausência de doenças ou traumas que comprometessem a mobilidade do sistema musculoesquelético. Este trabalho foi devidamente analisado e aprovado pela comissão de ética do Hospital das Clínicas da Faculdade de Medicina da Universidade de São Paulo. Todos os participantes que concordaram em fazer parte deste estudo foram devidamente esclarecidos e assinaram o temo de consentimento padrão daquela comissão.

A mobilidade do tornozelo e pé foi obtida pela goniometria. Foram medidas as amplitudes de movimento passivas máxima da dorsiflexão e flexão plantar do tornozelo e da extensão das cinco articulações metatarso-falângicas. Utilizouse um goniômetro universal e um goniômetro de dedos. Para a realização das medidas da dorsiflexão e da flexão plantar, os participantes permaneceram sentados em uma maca tipo divã, com quadril, joelho e tornozelo em $90^{\circ}$ e o goniômetro posicionado na face lateral do pé, um de seus braços acompanhando a linha da fíbula e o outro o quinto metatarso. A posição inicial para as medidas foi o pé a $90^{\circ}$ graus em relação à perna, a partir da qual se mediu a dorsiflexão, e a flexão plantar passivas máximas ${ }^{16}$. Considerando que nas articulações dos dedos 2, 3 e 4 não é possível posicionar o goniômetro como nas demais articulações, optou-se por utilizar o goniômetro de dedos. Nestas medidas os participantes permaneceram sentados no divã, o goniômetro foi posicionado na face plantar dos pés, sob cada dedo, com um dos braços do goniômetro seguindo o metatarso e o outro acompanhou o deslocamento do dedo medido. Para a obtenção de apenas um valor para a amplitude da extensão dos cinco dedos, calculou-se uma média aritmética sendo este o valor considerado para a extensão dos dedos. Por se tratar de uma articulação que tem grande participação na marcha, a extensão do hálux também foi considerada separadamente. Todas as medidas foram realizadas pelo mesmo avaliador. 
Para a obtenção do pico da força vertical de reação do solo (FRS), foi utilizado o sistema FSCAN (versão3. 816), que utiliza palmilhas flexíveis dotada de sensores de carga, em toda a superfície, totalizando 960. As palmilhas são conectadas a transdutores fixados na perna dos indivíduos por meio de braçadeiras, dos quais partiram cabos coaxiais conectando as palmilhas ao microcomputador. O programa específico do sistema registra e analisa os dados colhidos. As palmilhas são recortadas de acordo com o tamanho do pé e inseridas no calçado. Foram utilizadas o mesmo tipo de meia em todos os participantes, o modelo foi a meia social masculina 90\% poliéster, cedidas pelo laboratório, bem como o mesmo tipo de calçado para todos, o padrão foi o tênis modelo iate, o próprio laboratório dispõe de um par nas diversas numerações variando do 34 ao 45. Todos os tênis eram novos e utilizados apenas para exames. Cada participante teve seu calçado adequado à sua numeração e grau de conforto. Após a escolha do calçado cada participante pode deambular pela pista de exames para se familiarizar com o calçado e com o local de exames. Uma vez conectados ao sistema, e antes de cada exame, foi realizada a calibração utilizando o valor do peso corporal. Uma palmilha nova foi usada a cada exame. Todos os participantes foram orientados a caminhar por quatro vezes em uma pista de 12 metros em velocidade confortável de marcha. Durante esta caminhada o examinador dava o comando no teclado do computador, que iniciava a gravação da seqüência dos passos. Foi gravada uma seqüência de seis a oito passos. Cessada a coleta o programa traçou e exibiu na tela do computador, o gráfico da força vertical de reação do solo em função do tempo. Para evitar as interferências do início da marcha na pista e do final, foram selecionados apenas os quatro passos centrais. O valor do pico de força vertical em cada passo pôde ser obtido automaticamente segundo cada curva (passo) selecionada, calculou-se então a média aritmética. Para fins de normalização, o sistema foi programado para apresentar os valores da força vertical em kilograma-força para posteriormente ser calculada a porcentagem do peso corporal. Valor este considerado nas correlações.

A análise dos dados contou com o teste estatístico de Sperman para a verificação da correlação entre mobilidade e força. Considerando que foram medidos os pés direito e esquerdo, aplicou-se também o teste estatístico de Wilcoxon a fim de verificar a possibilidade de trabalhar com ambos os pés em um só grupo. Para observar se houve diferença entre os valores do grupo feminino e masculino aplicou-se o teste estatístico de Mann-Whitney. Para os testes foi considerado nível de significância quando $\mathrm{p}<0,05$.

\section{RESULTADOS}

Os valores médios obtidos em cada uma das medidas realizadas encontram-se dispostos na tabela 1 . Não houve diferença estatística entre os grupos quanto à idade. Não foi encontrada diferença entre os pés direito e esquerdo em todas as variáveis tanto no grupo masculino quanto feminino, condição que permitiu unir os valores obtidos dos pés direito e esquerdo totalizando 30 pés avaliados. Foi encontrada diferença na mobilidade de homens e mulheres, exceto para a dorsiflexão. Por esta razão os grupos foram estudados separadamente. Não houve diferença entre os sexos para a FRS. Quando foram correlacionados as medidas da goniometria e o pico da FRS, no grupo masculino foi encontrada correlação de Speraman significativa entre a FRS e a dorsiflexão (figura 1). No grupo feminino a correlação estatisticamente significante se deu entre a flexão plantar e a FRS (figura 2), entre a extensão do hálux e a FRS (figura 3) e entre a extensão dos dedos e a FRS (figura 4). Para as demais correlações não foi observado resultado estatisticamente significante.

Tabela 1. Valores médios e o desvio padrão da goniometria expressos em graus e dos valores normalizados da força vertical de reação do solo pelo peso corporal expressos em porcentagem.

\begin{tabular}{llllll}
\hline & N & \multicolumn{2}{c}{ Masculino } & \multicolumn{2}{c}{ Feminino } \\
& & Média & DP & Média & DP \\
\hline Dorsiflexão & 30 & $17,50^{\circ}$ & 4,50 & $19,16^{\circ}$ & 6,02 \\
Flexão Plantar & 30 & $41,83^{\circ} *$ & 9,04 & $52,33^{\circ} *$ & 14,18 \\
Extensão hálux & 30 & $67,50^{\circ} *$ & 11,35 & $73,16^{\circ} *$ & 8,35 \\
Extensão dedos & 30 & $67,83^{\circ} *$ & 11,36 & $74,25^{\circ} *$ & 7,65 \\
Força vertical (\%) & 30 & $88,61 \%$ & 20,46 & $89,33 \%$ & 13,20
\end{tabular}

$\mathrm{N}=$ número de pés (direito e esquerdo) DP = desvio-padrão(*) Diferença estatisticamente significante entre os sexos feminino e masculino (Mann-Whitney, $\mathrm{p}<0,05$ ).

\section{DISCUSSÃO}

A análise quantitativa da mobilidade dos pés é limitada, devido à complexidade de suas estruturas, bem como à limitação técnica dos recursos disponíveis. O número significativo de articulações apresentando-se em planos e eixos diferentes torna a adequação de equipamentos para medição ${ }^{17}$ e metodologias de estudo bastante escasso, principalmente na área clínica onde a avaliação da mobilidade dos pés é parte essencial do exame funcional. Para desenvolver adequadamente suas funções principalmente os eventos da marcha, incluindo os aspectos cinemáticos e cinéticos, o pé necessita do perfeito funcionamento de todas as suas articulações. Conhecer os efeitos do mau funcionamento destes parâmetros também é função do fisioterapeuta durante sua avaliação.

O presente trabalho foi desenvolvido dentro do Instituto de Ortopedia e Traumatologia da Universidade de São Paulo, 


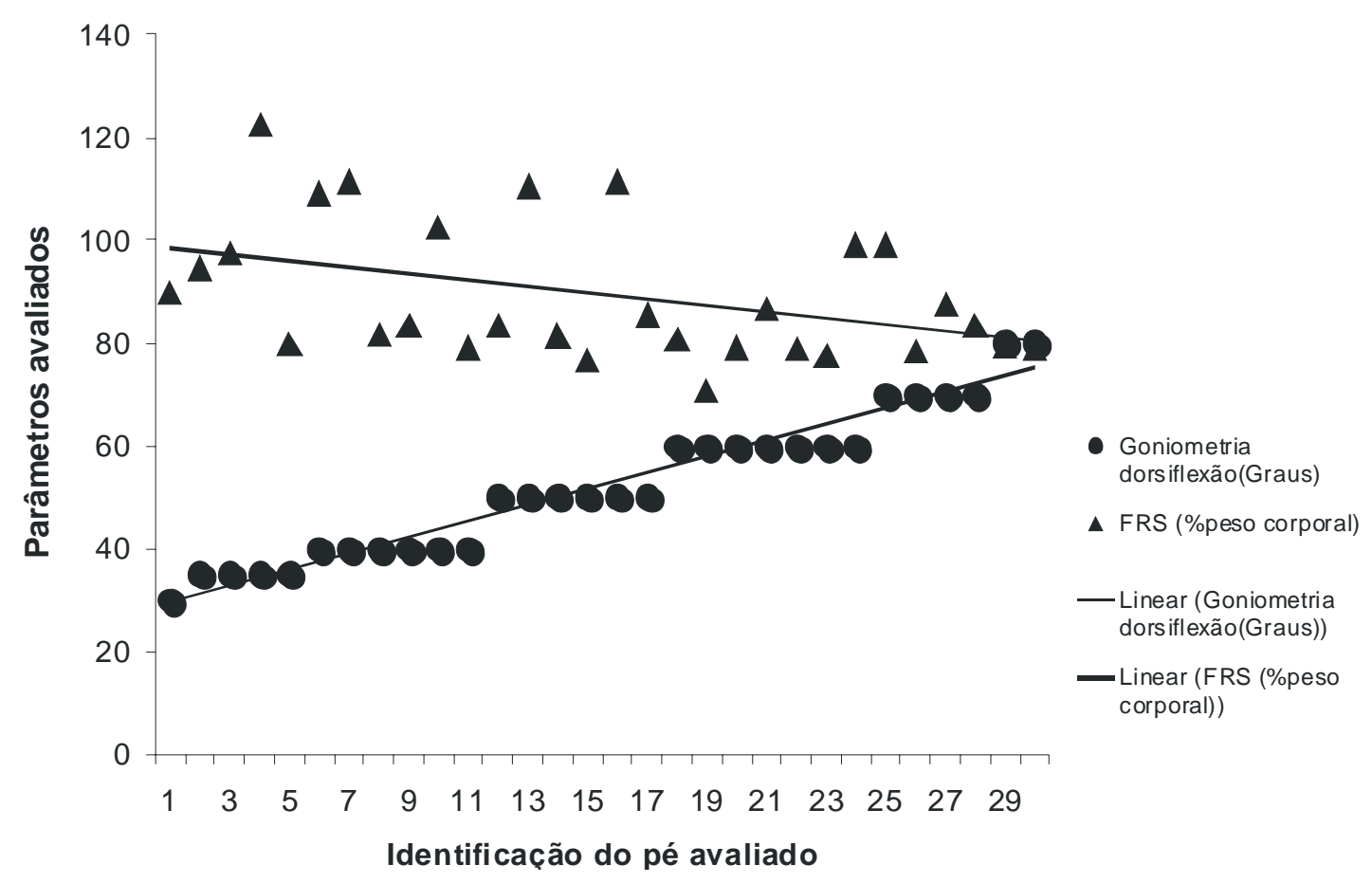

Correlação de Spearman significativa $(r=-0,399)$.

Figura 1. Distribuição dos valores da goniometria de dorsiflexão do tornozelo e força de reação do solo (FRS), no grupo masculino, com as respectivas linhas de tendência.

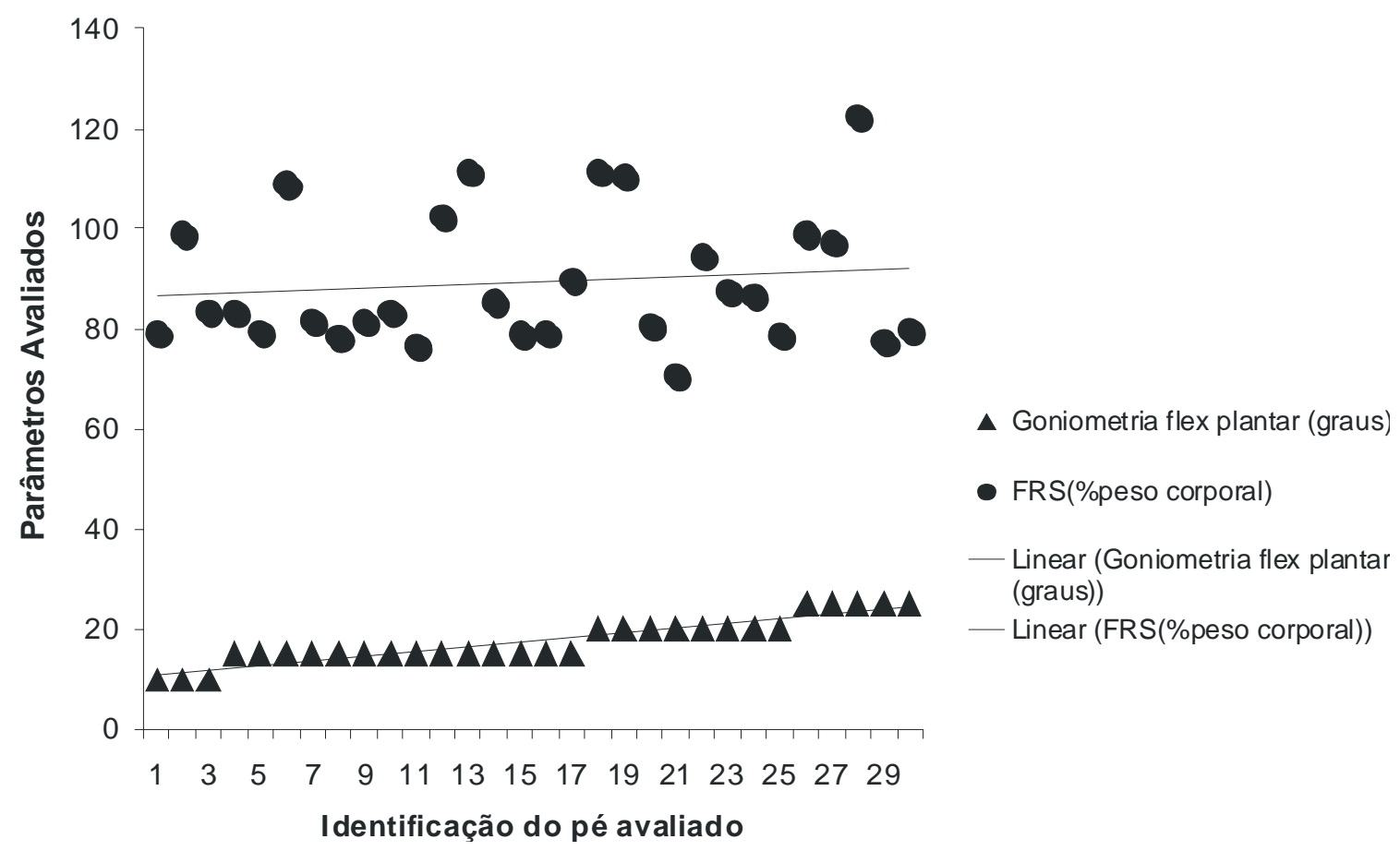

Correlação de Spearman significativa $(r=-0,411)$.

Figura 2. Distribuição dos valores da goniometria da flexão plantar e a força vertical (FRS), no grupo feminino, com as respectivas linhas de tendência. 


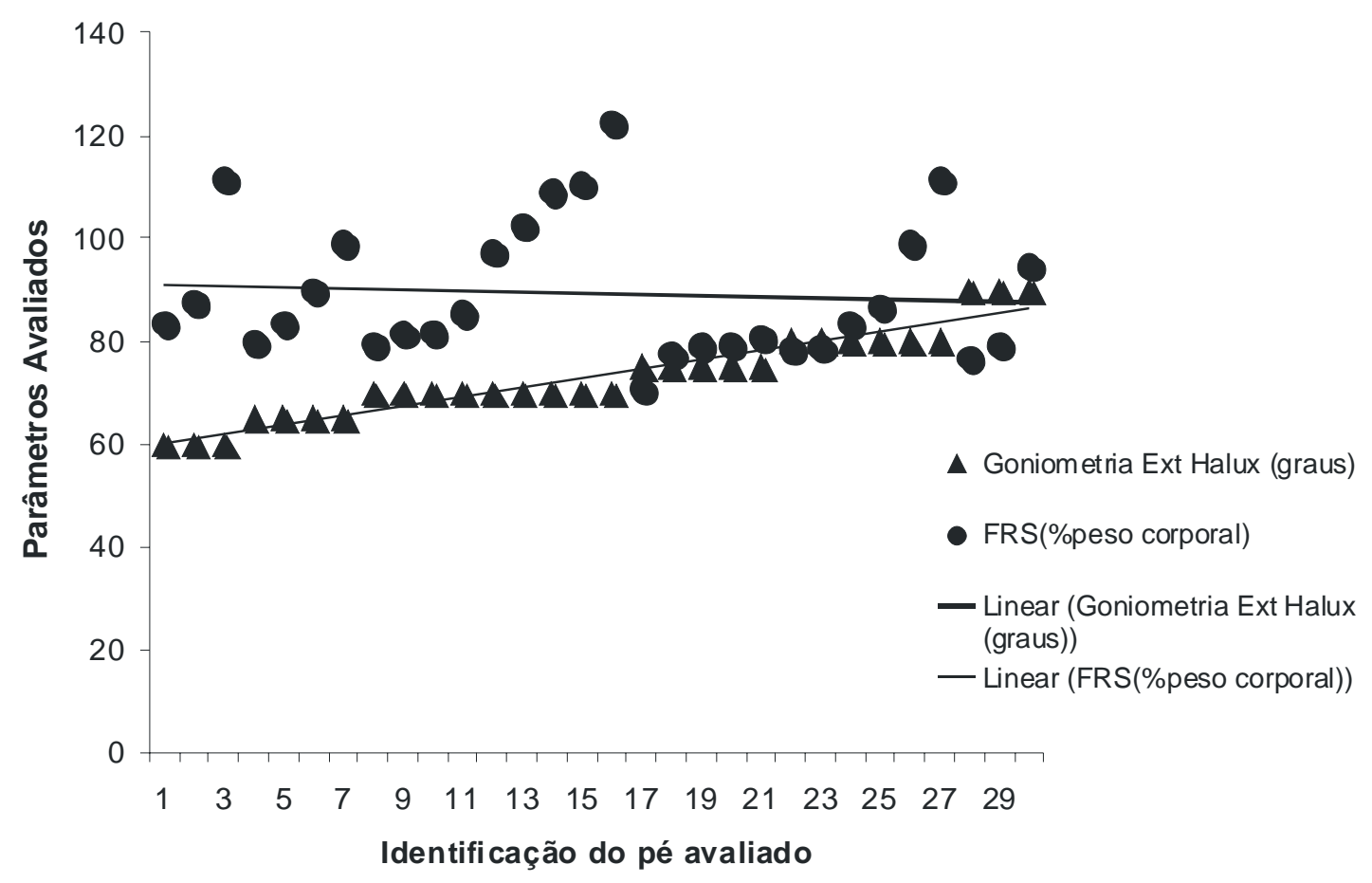

Correlação de Spearman significativa $(r=-0,386)$.

Figura 3. Distribuição dos valores da goniometria da extensão do hálux e a força vertical (FRS), no grupo feminino, com as respectivas linhas de tendência.

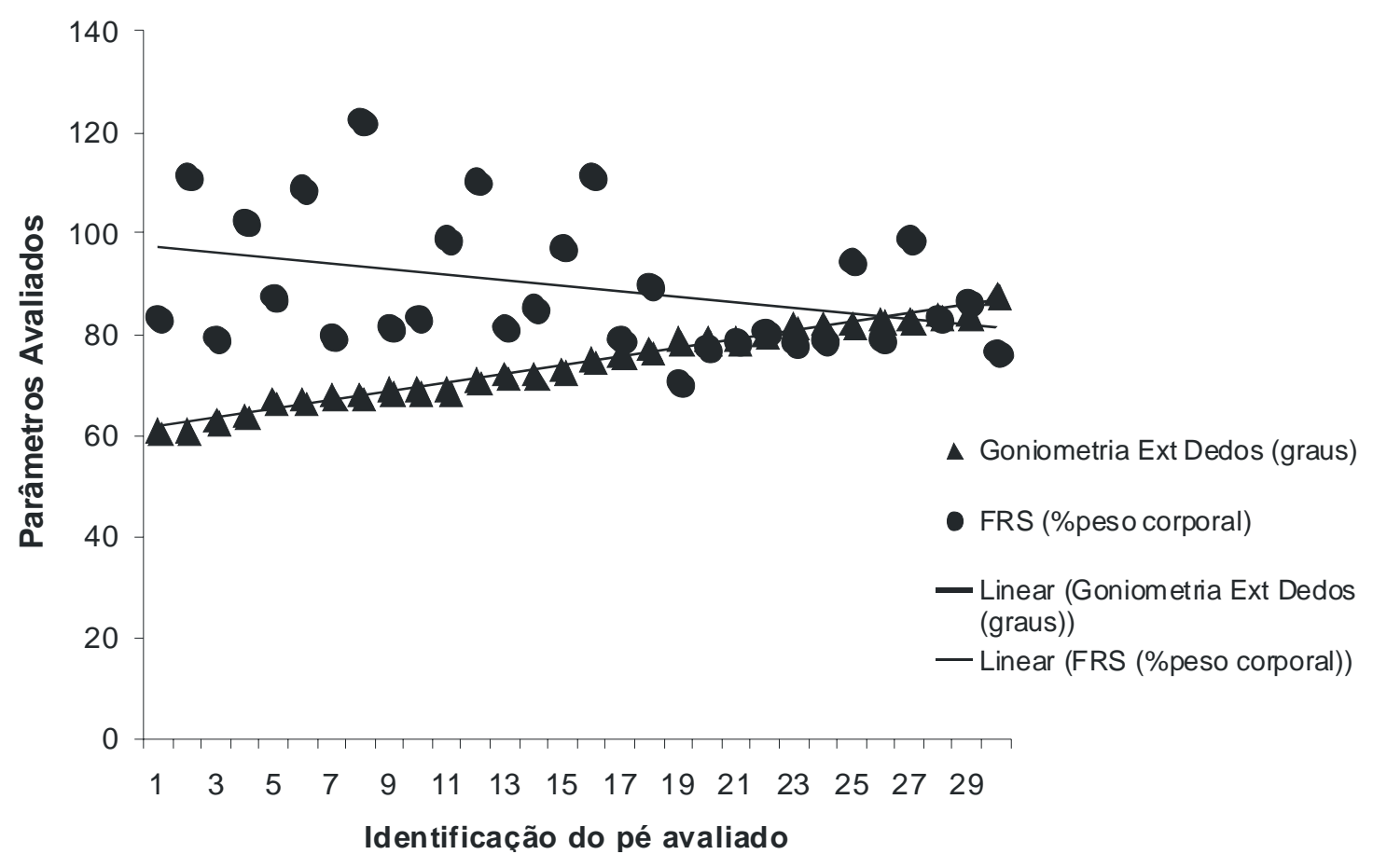

Correlação de Spearman significativa $(r=-0,452)$.

Figura 4. Distribuição dos valores da goniometria da extensão dos dedos e a força vertical (FRS), no grupo feminino com as respectivas linhas de tendência. 
cujo laboratório é voltado para análise da função do sistema musculoesquelético, considerando principalmente as aplicações clínicas. Sendo assim, a necessidade de investigar até que ponto a redução da mobilidade interfere nas forças geradas na marcha, surgiu da observação dos indivíduos com doenças já instaladas, onde a mobilidade se apresentava ausente ou reduzida. A literatura já aborda esta questão tendo como foco as lesões e deformidades que promovem as alterações da mobilidade ${ }^{7,18,20}$, sendo uma das principais áreas de estudo as alterações do pé diabético $0^{5,7,9,10,11,12,13}$. Considerando os objetivos e metodologia adotada nos trabalhos levantados na literatura, não foi observada a investigação direta da relação entre a mobilidade e a força vertical gerada durante a marcha ${ }^{8,15}$.

Empregou-se o sistema comercial FSCAN, destinado para análise das pressões plantares, por se tratar de um sistema simplificado com aplicabilidade clínica viável, que permite aos examinadores obterem dados quantitativos da marcha em tempo real e transportar suas informações para aplicabilidade clínica. Condição esta difícil de obter quando os indivíduos precisam ser encaminhados aos laboratórios fora dos centros de atendimento. Considerando a simplicidade do sistema e suas limitações, o estudo procurou se limitar a investigação dos indivíduos assintomáticos e a uma amostra com boa homogeneidade, principalmente quanto aos fatores de inclusão e a metodologia.

Foi observada homogeneidade entre as medidas dos pés direito e esquerdo (Wilcoxon), por esta razão os grupos passaram a ter um número de 30 pés. O mesmo não ocorreu com relação aos sexos (Mann-Whitney), desta forma trabalharam-se separadamente os sexos. As mulheres mostraram maior mobilidade articular que os homens. Todos os valores obtidos de amplitude de movimento articular se encontravam dentro dos parâmetros fisiológicos ${ }^{21,22}$, obtidos também de forma estática.

Os valores do pico da FRS foram normalizados pelo peso corporal para fins de comparação entre indivíduos ${ }^{24}$. Homens e mulheres apresentaram valores semelhantes da FRS, não houve diferença estatisticamente significante (MannWhitney).

Na relação entre a FRS e a mobilidade, foi encontrada no grupo feminino, uma correlação negativa estatisticamente significante entre a FRS e a flexão plantar, entre a FRS e a extensão dos dedos e entre a FRS e a extensão do hálux. No grupo masculino, foi obtida correlação negativa estatisticamente significante entre a FRS e a dorsiflexão. Os resultados estatísticos apresentaram valores negativos de correlação indicando que à medida que um dos parâmetros tende a aumentar o outro tende a diminuir. Autores como Veves et al. ${ }^{10}$ e Fernando et al. ${ }^{12}$ encontraram correlação entre a diminuição da mobilidade e o aumento dos valores da pressão plantar, entretanto não é possível uma comparação direta com nossos resultados uma vez que pressão e força são medidas distintas, nem sempre onde há aumento do valor da força haverá aumento da pressão. D’Ambrogi et al. ${ }^{24}$ estudaram entre outros parâmetros a mobilidade dos pés, a integral da força vertical pelo tempo e o tempo de aplicação desta força na superfície plantar, em indivíduos diabéticos. Os autores, entretanto, tiveram como objetivo comparar os dados entre os indivíduos com e sem diabetes e não fizeram a correlação dos dados dentro do mesmo grupo. Dois outros trabalhos investigaram os efeitos da diminuição da mobilidade gerada por cirurgias de artrodese, entretanto estes estudos também não correlacionam os valores da mobilidade com os da força vertical, seus estudos procuram associar a dinâmica dos movimentos com o comportamento da força vertical durante o desenrolar da marcha, nos pés com e sem a artrodese ${ }^{8,15}$. Estes estudos, mesmo sem realizar a correlação dos valores da mobilidade e da força vertical, também corroboram com a hipótese de que alterações na mobilidade dos pés trazem interferência nas das forças plantares.

Apesar dos resultados não se repetirem da mesma maneira nos homens e nas mulheres, foram encontrados resultados estatisticamente significantes em ambos, mostrando que a redução da mobilidade interfere na força vertical gerada na marcha, mesmo para pés considerados normais. Possivelmente esta condição ao longo do tempo pode ser a justificativa para alguns quadros de pés dolorosos. A mobilidade é um aspecto importante e precisa ser levado em consideração durante a avaliação funcional dos pés, e a sua preservação é essencial para o bom funcionamento da dinâmica dos mesmos e de todo membro inferior.

Recomendamos para estudos futuros que a metodologia seja reproduzida em um laboratório de análise do movimento onde os valores da mobilidade possam ser adquiridos de forma dinâmica e sincronizados com os valores da força de reação do solo. Uma vez estabelecidos parâmetros para o estudo dos pés, estas correlações poderão ser estendidas para as demais articulações a fim de analisar o efeito que uma lesão nos pés poderá causar nas outras articulações. As limitações deste trabalho dizem respeito à tecnologia empregada, onde a mesma se destina principalmente a estudos clínicos, e à dificuldade em se estudar dinamicamente estrutura de tamanho reduzido e que se movimentam em planos diversos como as articulações dos pés.

\section{CONCLUSÃO}

Existe correlação negativa entre a mobilidade do pé e tornozelo e o pico de força vertical de reação do solo em ambos os sexos.

Agradecimentos: Laboratório de Estudo do Movimento do IOT-USP e Capes. 


\section{REFERÊNCIAS BIBLIOGRÁFICAS}

1. Kitaoka HB, Lundemberg A, Luo ZP, Na Kai-Nam. Kinematics of the normal arch of the foot and ankle under physiologic loading. Foot Ankle 1995; 16: 492-99.

2. Donaghue VM, Veves A. Foot pressure measurement. Orthop. Phys. Ther. Clin. North Am. 1997; 6: 1509-16.

3. Mann RA, Hagy JL. The function of the toes in walking, jogging and running. Clin. Orthop 1979; 142: 24-9.

4. Mueller MJ, Diamond JE, Delitto A, Sinacore DR. Insensitive, limited joint mobility, and plantar ulcers in patients with diabetes mellitus. Phys. Ther 1989; 69: 453-62.

5. Muller FB, Lamoreaux L. Significance of free dorsoflexion or the toes in walking. Acta. Orthop Scand 1979; 50:471-79.

6. Hughes J, Clark P, Klenerman L. The importance of the toe in walking. J. Bone Joint Surg[BR] 1990; 72: 245-51.

7. Birke JA, Cornwall MW, Jackson M. Relationship between hallux limitus and ulceratios of the great toe. J. Orthop. Sport Phys. Ther 1988; 10:172-76.

8. Beyaert C, Sirveaux F, Paysant J, Mole D, Andre JM. The effect of tibio-talar arthrodesis on foot kinematics and ground reaction force progression during walking. Gait Posture 2004; 20:84-91.

9. Delbridge L, Perry S, Marr S, Arnold N, Yue DK, Turtle JR, et al. Limited joint mobility in the diabetic foot: relationship to neuropathy ulceration. Diabetic Med 1988; 5: 333-37.

10. Veves A, Sarnow MR, Guirini JM, Rosenblum BI, Iyons TE, Chrzan JS, et al. Differences in joint mobility and foot pressures between black and white diabetic patients. Diabetic Med 1995; $12: 585-89$

11. Birke JA, Franks DB, Foto JG. Firt ray limitation, pressure, and ulceration of first metarsal head in diabetes mellitus. Foot Ankle 1995; 16:277-84.

12. Fernando DJS, Masson EA, Veves A, Boulton AJM. Relationship of limited joint mobility to abnormal foot pressures and diabetic foot ulceration. Diabetes Care 1999; 14: 8-11.
13. Sarnow MR, Veves A, Giurini JM, Rosenblum BI, Chzan JS, Habershaw GM. In-shoe foot pressure measurements in diabetic patients with at-risk feet and healthy subjects. Diabetes Care 1994; 17:1002-06.

14. Sneyers CJL, Lysens R, Feys H, Andries R. Influence of malalidnment of the feet on the plantar pressures pattern in running. Foot Ankle 1995; 16: 624-32.

15. Galois L, Girard D, Martinet N, Delagoutte JP, Mainard D. Optoeletronic gait analysis after metatarsophalangeal arthrodesis of the hallux: fifteen cases. Rev Chir Orthop Reparatrice Appar Mot 2006; 92:52-9.

16. Marques AP. Manual de Goniometria. São Paulo: Editora Manole Ltda; 1979.

17. Razeghi M, Batt ME. Biomechanical analysis of the effect of orthotic shoe inserts. A review of the literature. Sports Med 2000; 29:425-38.

18. Minns RJ, Craxford AD. Pressures under the forefoot in rheumatoid arthritis. Clin. Orthop 1984; 187:235-42.

19. Carson MC, Hrrington ME, Thompson N, O’Connor JJ, Theologis TN. Kinematics analysis of a multi-segment foot model for research and clinical applications: a repeatability analysis. J. Biomech 2001; 34: 1299-1307.

20. Rodgers, MM. Dynamic biomechanics of the normal foot and ankle during walking and running. Phys. Ther 1988; 68:1822-30.

21. Teixeira LF, Olney SJ. Anatomia funcional e biomecânica das articulações do tornozelo, subtalar e médio-társica. Rev. Fisioter. Univ. São Paulo 1997; 4: 50-65.

22. Oatis CA. Biomechanics of the foot and ankle under static conditions. Phys Ther 1988; 68:1815-21.

23. Oliveira GS, Greve JMD, Imamura M, Bolliger R. Interpretação das variáveis quantitativas da baropodometria computadorizada em indivíduos normais. Revista do Hospital das Clínicas da Faculdade de Medicina de São Paulo 1998; 53: 16-20.

24. D’Ambrogi E, Giacomozzi C, Macellari V, Uccioli L. Abnormal foot function in diabetic patients: the altered onset of windlass mechanism. Diabet Med 2005; 22:1713-9 